IP/BBSR/2004-6

\title{
Supersymmetric Quantum Mechanics on Non-Commutative Plane
}

\author{
E. Harikumar*, V. Sunil Kumar ${ }^{\dagger}$ and Avinash Khare ${ }^{\ddagger}$ \\ Institute of Physics, \\ Sachivalaya Marg, Bhubaneswar \\ Orissa, INDIA-751 005
}

October 29, 2018

\begin{abstract}
We study the Pauli equation on non-commutative plane. It is shown that the Supersymmetry algebra holds to all orders in the noncommutative parameter $\theta$ in case the gyro-magnetic ratio $g$ is 2 . Using Seiberg-Witten map, the first order in $\theta$ correction to the spectrum is obtained in the case of uniform magnetic field. We find that the eigenstates in the non-commutative case are identical to the commutative case provided the magnetic field $B$ is everywhere replaced by $B(1+B \theta)$.
\end{abstract}

PACS Numbers: 11.10.Nx, 02.40.Gh, 11.30.Pb

Keywords: non-commutative plane, Supersymmetry, Pauli equation

\footnotetext{
*hari@iopb.res.in

${ }^{\dagger}$ sunil@iopb.res.in

${ }^{\ddagger}$ khare@iopb.res.in
} 


\section{Introduction}

Different aspects of the field theories formulated on non-commutative spaces [1] have been studied in the last few years. These studies are motivated by the developments in string theories and also from the attempts to understand the renormalization program [2]. Studies in quantum gravity and matrix models [3] brought out interesting aspects of non-commutative spaces. Formulation of gauge theories on non-commutative space shows many interesting features [4] and have been widely investigated [5]. For example, the problem of spin 1/2 particles in the constant electro-magnetic background has been analyzed $[6,7]$ in the context of non-commutative QED. In particular, in [6] Schwinger's world-line formulation has been worked out while in [7] a direct solution of Dirac equation has been studied.It is interesting to note that in non-commutative QED, particle pair production is unaltered at the tree level but have nontrivial effect at one loop level[7]. Further, as shown in [8], this effect is enhanced if one considers correction to $g-2$ in such a theory.

In the context of Non-locality introduced by the non-commutativity of the space leads to the mixing of UV and IR divergences in field theories formulated on non-commutative spaces [9]. The UV/IR mixing crucially affects the renormalisability of the theory $[9,10]$. Recently supersymmetric models on non-commutative spaces have been constructed and the renormalisability of these model have been studied [11].

Many interesting quantum mechanical problems on non-commutative (NC) spaces have been investigated and the effect of non-commutativity on the observables has been analyzed [12]. In particular, the Landau problem has been formulated and studied extensively on non-commutative settings [12]. But these results seems to differ from one another and further, naively different gauge choices seen to give different results. Recently using Seiberg-Witten map $[2,13]$, quantum Hall effect problem on non-commutative plane has been studied [14]. Starting from the action for the Schrödinger field coupled to $U(1)$ potential on non-commutative plane and using Seiberg-Witten map (to the leading order in $\theta$ ), equivalent commutative action for the case of uniform magnetic field was obtained [14]. The corresponding Schrödinger equation was then derived and the gauge independence of the physical observables was shown.

Recently renormalisability of non-commutative supersymmetric models which do not involve gauge fields have been analyzed and it has been argued 
that supersymmetric gauge theories on non-commutative spaces have better renormalisability [11]. However so far as we are aware of supersymmetric gauge theory models in NC settings have not been investigated so far. The purpose of this paper is to take first step in this direction. In this paper, we study the problem of a charged particle moving in the non-commutative plane with perpendicular magnetic field. Corresponding commutative problem, Pauli equation, is known to have supersymmetry [15]. We show that the non-commutative generalization of Pauli equation has supersymmetry to all orders in the non-commutative parameter $\theta$ and this conclusion is valid irrespective of whether the magnetic field is uniform or not. Using SeibergWitten map $[2,13]$ we obtain first order in $\theta$ correction to the spectrum in the case of uniform magnetic field and explicitly demonstrate the gauge independence but $\theta$ dependence of the spectrum. In section 2 we briefly present the essential features of non-commutative $U(1)$ gauge theory and the Seiberg-Witten map. In section 3, we formulate the Pauli equation in noncommutative settings and show to all orders in $\theta$ that the supersymmetry algebra is valid. The exact spectrum valid to first order in $\theta$, is explicitly obtained in Landau and symmetric gauges and it is shown that the spectrum is the same as that in the commutative case provided the uniform magnetic field $B$ is replaced everywhere by $B(1+B \theta)$. We present concluding remarks in section 4.

\section{Non-commutative gauge theory}

The non-commutative(NC) plane is defined by the co-ordinates $\hat{x}_{i}$ satisfying

$$
\left[\hat{x}_{i}, \hat{x}_{j}\right]=i \epsilon_{i j} \theta, \quad i, j=1,2 .
$$

In the limit $\theta \rightarrow 0$, we recover commutative plane and one requires the $\mathrm{NC}$ theory to reduce to usual commutative theory in this limit. It is well known that the functions on NC space can be obtained using Moyal-Weyl correspondence. Corresponding to each function $f(x)$ on commutative space one associate a function $\hat{F}(\hat{x})$ as

$$
\hat{F}(\hat{x})=\int d k d x \exp ^{i k(\hat{x}-x)} f(x),
$$

on NC space. On NC spaces the multiplication rule is modified and the new rule, the star product, is defined as

$$
f(x) * g(x)=\left.\exp ^{\frac{i}{2} \theta^{i j} \partial_{i}^{x} \partial_{j}^{y}} f(x) g(y)\right|_{x=y} .
$$


The gauge transformation of fields on NC space gets modified because of this new multiplication rule and hence the structure of the gauge theory is much different from the commutative theory [4]. The non-commutative $U(1)$ theory is invariant under the $*$ gauge transformations

$$
\begin{aligned}
\hat{\Psi}(x) & \rightarrow \hat{U}(x) * \hat{\Psi}(x) \\
\hat{A}_{i} & \rightarrow \hat{U}(x) * \hat{A}_{i} * \hat{U}(x)^{\dagger}+i \hat{U}(x) * \partial_{i} \hat{U}^{\dagger} \\
, &
\end{aligned}
$$

with $\hat{U} * \hat{U}^{\dagger}=1=\hat{U}^{\dagger} * \hat{U}$ and the action of the covariant derivative is $D_{i} \hat{f}=\partial_{i} \hat{f}+i\left[\hat{A}_{i}, \hat{f}\right]_{*}$. The $\hat{U}(1)_{*}$ invariant field strength is given as

$$
\hat{F}_{i j}=\partial_{i} \hat{A}_{j}-\partial_{j} \hat{A}_{i}-i\left[\hat{A}_{i}, \hat{A}_{j}\right]_{*}
$$

and under $U(1)_{*}$ transformation this field strength transforms covariantly,i.e.,

$$
\hat{F}_{i j} \rightarrow \hat{U} * \hat{F}_{i j} * \hat{U}^{\dagger} .
$$

Unlike the commutative $U(1)$ case, in the $\mathrm{NC} U(1)$ case the field strength is in general not gauge invariant; only the integrated expressions like action are gauge invariant. The Seiberg-Witten map [2] allows us to map the gauge covariant objects like $\hat{F}_{i j}$ in terms of gauge invariant objects in the commutative space. This map allows us to express the $\mathrm{NC}$ gauge theory in terms of the commutative gauge fields but with the explicit $\theta$ dependence which behaves as a background field. Thus after this mapping, the usual calculational tools of commutative theory can be applied.

The Seiberg-Witten (SW) map is obtained by demanding that the ordinary gauge potentials (living in the commutative space) which are connected by a gauge transformation are mapped to $\mathrm{NC}$ potentials which are connected by the corresponding $\mathrm{NC}$ gauge transformation. This requirement stated as

$$
\hat{A}(A)+\delta_{\hat{\lambda}} \hat{A}(A)=\hat{A}(A+\partial \lambda),
$$

gives the equation

$$
\hat{A}_{i}^{\prime}(A+\partial \lambda)-\hat{A}_{i}(A)-\partial_{i} \hat{\lambda}=-\epsilon^{k l} \theta \partial_{k} \lambda \partial_{l} A_{i} .
$$

Solving this equation, to the order $\theta$ gives the well-known SW map

$$
\begin{aligned}
\hat{A}_{i} & =A_{i}-\frac{1}{2} \epsilon^{k l} \theta A_{k}\left(\partial_{l} A_{i}+F_{l i}\right), \\
\hat{\lambda} & =\lambda+\frac{1}{2} \epsilon^{k l} \theta \partial_{k} \lambda A_{l},
\end{aligned}
$$


and for the matter field similar requirement results

$$
\hat{\psi}=\psi-\frac{1}{2} \epsilon^{k l} \theta A_{k} \partial_{l} \psi .
$$

From Eqn. (9) it follows

$$
\hat{F}_{i j}=F_{i j}+\epsilon^{k l} \theta\left(F_{i k} F_{j l}-A_{k} \partial_{l} F_{i j}\right) .
$$

Under the $U(1)$ transformation of the commutative gauge potential $A_{i} \rightarrow$ $A_{i}+\partial_{i} \lambda$, using Eqns. $(9,10,12)$ we get

$$
\hat{\delta}_{\hat{\lambda}} \hat{F}_{i j}=-\epsilon^{k l} \partial_{k} \lambda \partial_{l} F_{i j},
$$

where $F_{i j}$ is the usual commutative Maxwell field strength, thereby demonstrating the non invariance of $\mathrm{NC}$ field strength under $U(1)_{*}$ transformation except in the special case of uniform field strength. In this special case one can show that the exact relationship between $\hat{B}$ and $B$ (valid to all finite orders in $\theta)$ is [2]

$$
\hat{B}=\frac{B}{(1-B \theta)}
$$

where $\hat{B}=\hat{F}_{12}$. Note that this relationship is obviously not valid in case $B \theta=1$. We might add that there is an alternative approach using covariant coordinates [16], and Seiberg-Witten map can also be derived in that formalism.

\section{$3 \quad$ Pauli Hamiltonian in $R_{\theta}^{2}$}

In this section we study the problem of a charged particle moving in noncommutative plane $\left(R_{\theta}^{2}\right)$ with magnetic field in the perpendicular direction of the plane. The corresponding commutative problem is known to have supersymmetry [15]. We first briefly sketch the commutative supersymmetric problem. The Hamiltonian describing the system is given by $(\hbar=m=1)$

$$
H=\frac{1}{2}\left[-\left(\partial_{i}+i A_{i}\right)^{2}+\frac{g}{2} B \sigma_{3}\right],
$$

where $i=1,2$ and $B=F_{12}$. This problem is known to have supersymmetry (SUSY) in case the gyro-magnetic ratio, $g$, is 2 . In particular, in this case 
the two hermitian supercharges are

$$
\begin{aligned}
Q_{1} & =\frac{1}{\sqrt{2}}\left[i\left(\partial_{y}+i A_{y}\right) \sigma_{1}-i\left(\partial_{x}+i A_{x}\right) \sigma_{2}\right] \\
Q_{2} & =\frac{1}{\sqrt{2}}\left[-i\left(\left(\partial_{x}+i A_{x}\right) \sigma_{1}-i\left(\partial_{y}+i A_{y}\right) \sigma_{2}\right] .\right.
\end{aligned}
$$

where $\sigma_{i}$ are Pauli matrices. In terms of these, the complex supercharge is defined to be

$$
Q=-\frac{i}{2}\left(Q_{1}-i Q_{2}\right)
$$

It is straight forward to see that the SUSY algebra

$$
Q^{2}=0, \quad\left\{Q, Q^{\dagger}\right\}=H, \quad[H, Q]=0=\left[H, Q^{\dagger}\right]
$$

is satisfied where $H$ is given in Eqn. (15) with $g$ being 2 .

In this section we study generalization of this problem to $\mathrm{NC}$ plane. In the NC generalization of quantum mechanical problems as well as that of field theories it is known that one can either work directly with NC variables or work with usual commutative variables but with the usual products modified to star product $[12,17]$. The 2 dimensional central field problem, Landau problem etc have been analyzed using the second approach recently. In these papers the effect of star product has been absorbed in a momentum dependent shift of the co-ordinates. In symmetric gauge, the Landau problem has been shown to be equivalent to NC central field problem using this method [12]. But naively this approach seems to give gauge dependent answers. In [14] the NC Hall effect problem has been studied using the first approach and by employing the SW map the NC action was re-expressed in terms of the commutative variables and $\theta$ and the effect of non-commutativity was studied. We feel this approach is better suited to study problems involving gauge potential and we adopt this method in this paper.

We start with the Hamiltonian expressed in terms of the $\mathrm{NC}$ variables

$$
\begin{aligned}
\hat{H} & =\frac{1}{2}\left[-\left(\partial_{i}+i \hat{A}_{i}\right)^{2}+\frac{g}{2} \hat{B}_{3} \sigma_{3}\right] \\
& =\left(\begin{array}{cc}
\hat{H}_{2} & 0 \\
0 & \hat{H}_{1}
\end{array}\right)
\end{aligned}
$$

where hatted variables live on the NC plane and $\hat{B}=\frac{1}{2} \epsilon_{i j} \hat{F}_{i j}$ with $\hat{F}_{i j}$ given 
in Eqn. (5). The NC supercharges are

$$
\begin{gathered}
\hat{Q}_{1}=\frac{1}{\sqrt{2}}\left(\begin{array}{cc}
0 & -\left(\partial_{x}+i \hat{A}_{x}\right)+i\left(\partial_{y}+i \hat{A}_{y}\right) \\
\left(\partial_{x}+i \hat{A}_{x}\right)+i\left(\partial_{y}+i \hat{A}_{y}\right) & 0
\end{array}\right) \\
\hat{Q}_{2}=\frac{1}{\sqrt{2}}\left(\begin{array}{cc}
0 & -i\left(\partial_{x}+i \hat{A}_{x}\right)-\left(\partial_{y}+i \hat{A}_{y}\right) \\
-i\left(\partial_{x}+i \hat{A}_{x}\right)+\left(\partial_{y}+i \hat{A}_{y}\right) & 0
\end{array}\right)
\end{gathered}
$$

From this we get the complex super charge (Eqn. 17) to be

$$
\begin{aligned}
\hat{Q} & =\left(\begin{array}{cc}
0 & \mathcal{A} \\
0 & 0
\end{array}\right) \\
\text { where } \mathcal{A} & =\frac{1}{\sqrt{2}}\left[i\left(\partial_{x}+i \hat{A}_{x}\right)+\left(\partial_{y}+i \hat{A}_{y}\right)\right] .
\end{aligned}
$$

It is straight forward to see that the SUSY algebra

$$
\{\hat{Q}, \hat{Q}\}_{*}=0, \quad\left\{\hat{Q}, \hat{Q}^{\dagger}\right\}_{*}=\hat{H},[\hat{H}, \hat{Q}]_{*}=0=\left[\hat{H}, \hat{Q}^{\dagger}\right]_{*}
$$

is satisfied with $H$ as given in Eqn. (19) and $g=2$. Note that this algebra is valid irrespective of whether the field strength is uniform or not and also irrespective of the value of the NC parameter $\theta$. Thus we have shown that in the NC settings, the Pauli equation can be casted in the supersymmetric form provided $g$ is again 2. This conclusion is valid to all orders in $\theta$.

Using Eqn. (21) and (22) the SUSY partner Hamiltonians can be expressed as

$$
\hat{H}_{1}=\mathcal{A}^{\dagger} \mathcal{A}, \quad \hat{H}_{2}=\mathcal{A} \mathcal{A}^{\dagger},
$$

and the ground state wave function of $\hat{H}_{1}$ obeys

$$
\mathcal{A} \Psi_{0}^{1}=0,
$$

where $\mathcal{A}$ is given by Eq. (22).

We shall now specialize to the case of uniform magnetic field and solve the NC Pauli equation in two different gauges. We obtain the spectrum valid to first order in $\theta$ explicitly in both the Landau and the symmetric gauges. In the commutative case, when the magnetic field is uniform, then in the Landau gauge the corresponding gauge potentials are given by

$$
A_{x}=-B y, \quad A_{y}=0,
$$


while in the symmetric gauge, they are given by

$$
A_{x}=-\frac{1}{2} B y, \quad A_{y}=\frac{1}{2} B x .
$$

These two gauges are connected by $U(1)$ gauge transformation $A_{i} \rightarrow A_{i}+\partial_{i} \lambda$ where the transformation parameter $\lambda$ is given by

$$
\lambda=-\frac{1}{2} B x y .
$$

The NC potential in a given gauge can be expressed in terms of the corresponding commutative potential and $\theta$. For example, in the Landau gauge, to order $\theta$, such a connection is easily obtained by using Eqn.(9) and one finds that

$$
\hat{A}_{x}=-B(1+B \theta) y, \quad \hat{A}_{y}=0 .
$$

On the other hand, using Eqns.(26,27,28 and 10) in Eqn.(8), the NC gauge potential in the symmetric gauge is given by

$$
\hat{A}_{x}=-\frac{1}{2} B(1+B \theta) y, \quad \hat{A}_{y}=\frac{1}{2} B(1+B \theta) x .
$$

Using Eqns. (12),(13) and (14) it is clear that corresponding to the commutative magnetic field $B$, the NC magnetic field $\hat{B}$, to order $\theta$ is given by

$$
\hat{B}=B(1+B \theta) .
$$

Using Eqs. (21), (24) and (28) one can now immediately obtain the ground state wave function of $\tilde{H}$ in the Landau gauge. We find that

$$
\psi_{0}^{1}=\exp (i k x) \exp \left[-\frac{\rho^{2}}{2 B(1+\theta B)}\right]
$$

where $\rho=(B y-k)$ and $k$ being any real number. Similarly, using the SW map, in the Landau gauge, the NC Pauli equation $\hat{H} \psi=E \psi$ takes the form

$$
\left[-\partial_{y}^{2}-\left(\partial_{x}-i(1+B \theta) B y\right)^{2}+B(1+B \theta) \sigma\right] \Psi=2 E \Psi
$$

where we have used Eqns. (28) and (30). Thus in this gauge, effectively the problem has become an one dimensional one. With the redefinition $B^{\prime}=$ 
$B(1+B \theta)$, we get the same equation as in the commutative space [15] and hence the spectrum is given by

$$
E_{n}=\left(n+\frac{1}{2}+\frac{1}{2} \sigma\right) B(1+B \theta), \quad \sigma= \pm 1 .
$$

Thus the energy eigenvalues, spectral spacing and density of states get a $\theta$ dependent modification. Further, as in the commutative case, the spectrum is again independent of $k$ (see Eqn. (31)). Thus the energy levels are continuously degenerate.

In the symmetric gauge, using Eqns. (29) and (30), the SW mapped Pauli equation becomes

$$
\left[-\left(\partial_{x}-\frac{i}{2} B^{\prime} y\right)^{2}-\left(\partial_{y}+i \frac{i}{2} B^{\prime} x\right)^{2}+B^{\prime} \sigma\right] \Psi=2 E \Psi
$$

where $B^{\prime}=B(1+B \theta)$. As in the previous case, the above equation has exactly the same form as in the commutative symmetric gauge and hence the spectrum is

$$
E_{n}=\left(n+\frac{1}{2}+\frac{1}{2} \sigma+m+|m|\right) B(1+B \theta), \quad \sigma= \pm 1, \quad m=0, \pm 1, \pm 2, . . .
$$

Here also the energy eigenvalues, the spectral spacing as well the density of states are modified due to the $\mathrm{NC}$ parameter $\theta$ while the degeneracy is exactly the same as in the commutative case. In particular, we see that all the energy levels including the ground state are infinite-fold degenerate.

Thus to the first order in $\theta$, the spectrum while gauge invariant, is affected by the non-commutativity and the NC parameter $\theta$ appears explicitly in the energy eigenvalue expression. We see from Eqns. (33) and (35) that the ground state energy for $\hat{H}_{1}$ is zero in both gauges and thus to first order in $\theta$, SUSY continues to remain unbroken in the NC plane.

Before finishing this note, it is worth pointing out that in case we assume that even in the $\mathrm{NC}$ case, in the Landau gauge, $\hat{A}_{y}=0$, then using the exact SW map and using the exact relationship between $\hat{B}$ and $B$ as given by Eq.(14), it is easy to show that the exact relationship between $\hat{A}_{x}$ and $A_{x}$ in the Landau gauge is given by

$$
\hat{A}_{x}=\frac{A_{x}}{(1-B \theta)}=-\frac{B y}{(1-B \theta)}, \quad \hat{A}_{y}=0 .
$$


Hence it is now easy to show that to all orders in $\theta$, the exact spectrum is given by

$$
E_{n}=\left(n+\frac{1}{2}+\frac{1}{2} \sigma\right) \hat{B}, \quad \sigma= \pm 1
$$

where $\hat{B}$ is as given in Eqn.(14). The SUSY remains unbroken to all orders in $\theta$.

\section{Conclusion}

In this paper we have formulated and studied the non-commutative generalization of the Pauli equation and have shown how even that can be put in a supersymmetric setting. We have shown to all orders in $\theta$ that the SUSY algebra continues to hold in the NC case for $g=2$. Using SW map, we have obtained the equivalent Hamiltonian, to first order in $\theta$, in terms of commutative variables and NC parameter $\theta$. Using this SW mapped Hamiltonian, we have obtained the energy eigenvalues in two different gauge choices in case the magnetic field is uniform. The spectrum gets affected by the noncommutativity and to the first order in $\theta$ we have shown that all the energy eigenvalues are scaled by the factor of $1+B \theta$. We have also shown that, to order $\theta$, the SUSY remains unbroken in the NC plane. The spectrum is infinitely degenerate as in the commutative plane and interestingly the degeneracy does not depend on $\theta$ though the spectral spacing does. Finally, assuming that even in the NC Landau gauge $\hat{A}_{y}=0$, we have obtained the spectrum which is valid to all orders in $\theta$.

We have used the SW map to re-express to order $\theta$, the NC Hamiltonian in terms of commutative potential $A_{i}$ and $\theta$. It has been claimed [18] that

in the the SW map the $\theta^{2}$ and higher order terms are not unique. Since the SUSY is satisfied to all orders in $\theta$, demanding the SUSY algebra order by order in $\theta$ may allow us to single out the preferred choice of the higher order $\theta$ terms in SW map.

Acknowledgements: One of us (EH) thank M. Sivakumar for useful discussions. 


\section{References}

[1] A. Connes, Noncommutative geometry, Academic Press, London, (1994).

[2] N. Seiberg and E. Witten, JHEP 09 (1999) 032.

[3] A. Connes, M. R. Douglas and A. Schwarz, JHEP 9802 (1998) 033.

[4] J. A. Harvey, 'Topology of the Gauge Group in Noncommutative Gauge Theory', hep-th/0105242; C. Sochichiu, 'Gauge Invariance and Noncommutativity', hep-th/0202014.

[5] M. R. Douglas and N. A. Nekrasov, Rev. Mod. Phys. 73 (2001) 977.

[6] L. Alvarez-Gaume and J.L.F. Barbon Int. J. Mod. Phys. A 16 (2001) 1123, hep-th/0006209.

[7] N. Chair and M. M. Sheikh-Jabbari, Phys. Lett. B504 (2001) 141, hepth/0009037.

[8] I.F. Riad and M. M. Sheikh-Jabbari, JHEP 0008 (2000) 045, 0008132.

[9] S. Minwalla, M. V. Raamsdonk, N. Seiberg, JHEP 0002 (2000) 020.

[10] I. Y. Areféva, D. M. Belov and A. S. Koshelev, Phys. Lett. B476 (2000) 431; S. Sarkar, B. Sathiapalan, JHEP 0105 (2001) 049, S. Sarkar, JHEP 0206 (2002) 003.

[11] H. O. Girotti, M. Gomes, V. O. Rivelles and A. J. da Silva, Nucl. Phys. B587 (2000) 299; ibid, Int. J. Mod. Phys. A 17 (2002) 1503.

[12] M. Chaichian, M. M. Sheikh-Jabbari and A. Tureanu, Phys. Rev. Lett. 86 (2001) 2716; J. Gamboa, M. Loewe, F. Mendez and J. C. Rojas, Mod. Phys. Lett. A16 (2001) 2075; ibid, Int. J. Mod. Phys. A 17 (2002) 2555; S. Bellucci and A. Nersessian, Phys. Lett. B542 (2002) 295-300. P. A. Horvathy, Annals Phys. 299 (2002) 128; H. R. Christiansen and F.A. Schaposnik, Phys. Rev. D65 (2002) 086005; V. P. Nair and A. P. Polychronakos, Phys. Lett. B505 (2001) 267; L. Mezincescu, 'Star Operation in Quantum Mechanics', hep-th/0007046. 
[13] A. A. Bichl, J. M. Grimstrup, L. Popp, M. Schweda and R. Wulkenhaar, 'Deformed QED via Seiberg-Witten Map',hep-th/0102103

[14] B. Chakraborty, S. Gangopadhyay and A.Saha, 'Quantum Hall effect on non-commutative plane through Seiberg-Witten map', hep-th/0312292.

[15] M. de Crombrugghe and V. Rittenberg, Ann. Phys. 151 (1983) 99; A. Khare and J. Maharana, Nucl. Phys. B244 (1984) 409; F. Cooper, A. Khare and U. Sukhatme, 'Supersymmetry in Quantum Mechanics', (World Scientific, 2001).

[16] J. Madore, S. Schraml, P. Schupp and J. Wess, Eur. Phys. J C16 (2000) 161; R. Jackiw, S.-Y. Pi, Phys. Rev. Lett. 88 (2002) 111603.

[17] A. Micu and M. M. Sheikh-Jabbari, JHEP 0101 (2001) 025.

[18] S. Fidanza, JHEP 0206 (2002) 016. 